\title{
Research Paper: The Effect of Strengthening and Balance Exercises on Static Stability Indices in Women With Knee Osteoarthritis; A Randomized Clinical Trial
}

\author{
Narges Jahantigh Akbari ${ }^{1}$ (D), Ahmad Reza Askary Ashtiani2* ${ }^{*}$, Salman Nouraisarjou ${ }^{3}$ (D) \\ 1. Physiotherapy Research Center, School of Rehabilitation, Shahid Beheshti University of Medical Sciences, Tehran, Iran. \\ 2. Health Promotion Research Center, Zahedan University of Medical Sciences, Zahedan, Iran. \\ 3. Department of Physical Therapy, School of Rehabilitation, Shahid Beheshti University of Medical Sciences, Tehran, Iran.
}

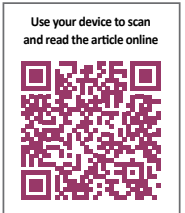

Citation: Jahantigh Akbari N, Askary Ashtiani AR, Nouraisarjou S. The Effect of Strengthening and Balance Exercises on Static Stability Indices in Women With Knee Osteoarthritis; A Randomized Clinical Trial. Iranian Rehabilitation Journal. 2019; 17(4):331-340. http://dx.doi.org/10.32598/irj.17.4.331

doi' http://dx.doi.org/10.32598/irj.17.4.331

Article info:

Received: 14 Apr 2019

Accepted: 09 Sep 2019

Available Online: 01 Dec 2019

Keywords:

Strengthening exercises, Knee osteoarthritis, Static stability biodex

\section{ABSTRACT}

Objectives: Knee osteoarthritis is one of the most common musculoskeletal problems that is associated with impaired balance. This study was conducted to determine the result of balance and strengthening training in static stability indices in females with knee osteoarthritis.

Methods: In this single-blind randomized controlled study, 13 patients were determined through the available sampling method and randomly assigned to strengthening exercises and balance exercises groups. Strengthening exercises were based on quadriceps strengthening and in the balance exercises group, it included balance exercises. Anteriorposterior, overall, and medial-lateral stability measures were performed by Biodex before and after the treatment in the static situation at the status of open and close eyes on one and two feet. The treatment was performed 15 sessions for 3 weeks. The data were analyzed by paired t-test and independent t-test.

Results: In strengthening exercises group, at the static status, overall and anterior-posterior stability index on two feet at the status of open eyes reduced from $1.26 \pm 0.82$ to $0.76 \pm 0.48$ $(\mathrm{P}=0.02)$ and from $0.87 \pm 0.53$ to $0.56 \pm 0.37(\mathrm{P}=0.02)$, respectively. In the balance exercise group, in the static status, overall lateral stability index on the involved foot at the status of close eyes reduced from $9.15 \pm 3.18$ to $7.53 \pm 2.81(\mathrm{P}=0.00)$ and from $8.27 \pm 3.32$ to $6.77 \pm 2.95$ $(\mathrm{P}=0.00)$, respectively. There was no significant difference between the groups $(\mathrm{P}>0.05)$.

Discussion: There was no difference between them regarding the impact on static stability indices. However, strengthening exercises caused improvement in the greater number of stability indices compared to those in the balance exercises group.

\footnotetext{
* Corresponding Author:

Ahmad Reza Askary Ashtiani, PhD.

Address: Health Promotion Research Center, Zahedan University of Medical Sciences, Zahedan, Iran.

Tel: +98 (915) 3415590

E-mail: Ahmadaskary@gmail.com
} 


\section{Highlights}

- Balance and strength exercises are effective in improving static stability indices.

- The long-term application of balance and strength exercises is more effective in improving static stability indices than its short-term use.

\section{Plain Language Summary}

Knee osteoarthritis is a chronic disease that is associated with functional limitations and pain. Neuromuscular disorders such as muscle weakness and proprioception disorder may lead to imbalance. The increased risk of falling and decreased motility in patients with knee osteoarthritis are attributed to equilibrium deficits. Exercise therapy, especially balance and strengthening exercises, is effective in the rehabilitation program of these patients. The present study aimed at investigating the effect of balance and strengthening exercises on static stability indices in females with knee osteoarthritis. The result shows that strengthening can improve more stability indices in females with knee osteoarthritis, especially in its long-term application.

\section{Introduction}

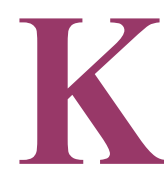

nee osteoarthritis is one of the most common musculoskeletal diseases in the knee joint [1]. It can lead to morphological and biological changes [2]. The disease becomes more prevalent with the increase in the individual's age and more increasingly higher rates have been reported in women [3]; $30 \%$ to $40 \%$ of the 65 -year-old women are usually diagnosed with the disorder and its prevalence rate is $72.6 \%$ in women $[3,4]$. Osteoarthritis is also realized as an important factor contributing to pain, debility, and low quality of life in oldaged people [5]. On the other hand, knee osteoarthritis causes a lot of problems in social, economic, psychological, and mental terms [6].

Osteoarthritis is a chronic and degenerative disease of the synovial joints adversely affecting the joint cartilage resulting in the gradual destruction of the cartilage and change in other tissues, including inflammation of the synovial membrane, muscular weakness, and osteogenesis reduction [7]. Pain and inflammation in patients with knee osteoarthritis make a problem in an individual's performance and neuromuscular control [8]. Therefore, individuals with knee osteoarthritis are predominantly complaining about proprioceptive disorders and inability in performing daily activities [6].

In addition to providing the joint with mechanical protection, the capsule and ligaments at the periphery of the knee joint also receive and transmit sensory feedbacks that bring about joint stability through creating muscular reflexes [9]. The performance of the quadriceps is disrupted in patients with knee osteoarthritis, following which the generation of force and proprioception will also be influenced [10]. The reduction in proprioception can result in the alteration of the muscular reflexes, the reduction of the muscle spindle sensitivity, and disorder in postural stability [11].

The proper performance of the protective neuromuscular mechanism depends on the appropriate sensorymotor functioning of the muscles at the periphery of the joint [9]. The disorder in the sensory functioning of the muscles causes a reduction in the proprioception and the disorder in the muscles' motor performance brings about muscular weakness [9]. The factors influencing the reduction in proprioception are the capsular-ligament laxity, inflammation, and pain [1]. The reduction in proprioception is a major cause resulting in the balance disorders in old-aged people [1]. Balance disorders in these old people have been reported to be in the form of an increase in the sway area and velocity and the speed of the postural sway [12].

There are numerous treatments suggested for increasing balance in patients with the osteoarthritic knee [6]. These treatments include weight loss, acupuncture, physiotherapy, movement therapy, Tai Chi, and other non-pharmaceutical treatments [13]. In a study, Fitzgerald et al. reported the significant roles of stability training in improving patients with knee osteoarthritis [6]. It is also stated in a study conducted by Kanda Chaipiny et al. in Thailand that physical training programs devised for individuals with knee osteoarthritis are simple and parallel to the elevation of the quadriceps strength. These exercises have been documented to have exerted useful 
effects [6]. In a study with the aim of comparison of the effects of strength training and neuromuscular control rehabilitation on the treatment indices of 30 patients (60to 65-year-old) with osteoarthritic knee, Shah Hosseini et al. reported a significant improvement in both of the groups in terms of balance indices [14]. It was shown in a systematic review study by Sumaiyah Mat et al. that physiotherapy interventions like strength training, Tai Chi, and aerobic exercises lead to an increase in balance in the individuals with knee osteoarthritis [6].

Postural stability disorders, pain, and disability resulting from the knee osteoarthritis, the social and economic symptoms, and the high prevalence rates of the disease in older adults, especially in women compared to the men, are the reasons why the researchers have studied the therapeutic aspects of the patients with such diseases [14]. The patients with knee osteoarthritis are engaged with many problems like debilities when walking, muscular atrophies, and knee instability [6]. Balance exercises along with strength training can play effective roles in the increase in knee stability [4]. Therefore, the present study aims at investigating the effect of balance and strength exercises on the static stability indices in women, who had knee problems, assuming that strength training and balance exercises will lead to increase in static stability and that the effect of the balance training on the static stability enhancement will be more pronounced than the other group.

\section{Methods}

\section{Study design}

This study was designed as a randomized controlled trial and single-blind in 2016 at Zahedan University of Medical Sciences. In this study, 26 women with osteoarthritis of the knee were equally divided into strengthening training and balance training groups. Participants in the study were blind about the information of the two groups. The treatment protocol was 5 sessions a week for 3 weeks [15]. The data were recorded before and after the treatment.

\section{Participants}

A total of 26 women were chosen through a simple random sampling method. The inclusion criteria were women, who had pain for 3 months or more [16], age range of 46 to 72 years [17], grade 1 to 4 based on Kellgren Lawrence Revised Grade Scale [18], joint morning stiffness resolved after 30 minutes [19], lack of history of injury [16], and lack of history of joint difficulty disor- ders (rheumatoid arthritis, osteoporosis, and neurological and musculoskeletal disorders) [16]. The exclusion criteria included having cardiovascular disease [19], getting physiotherapy for knee joint during 6 months [19], and having a history of knee joint operation [13], vestibular, severe visual, and peripheral neuropathy disorders [20]. After reading and signing a written informed consent form, they were included in the study protocol. This study was approved by the Scientific and Ethics Committee of Rehabilitation Department. This study was conducted in Zahedan, Iran. The rules of study were explaned and observed by the participants at all times.

\section{Study procedure}

\section{Assessment tools}

To ensure to meet the criteria, the patients were investigated. Tape meters were used to evaluate the height, digital scale were used to vevaluat body weight index (mass index); also, the Biodex Balance System was used to measure static stability indices.

The indices of overall stability, medial-lateral, and anterior-posterior were evaluated by Biodex SD (Balance System, Biodex, New York, SW 45-30D-E6N, Inc, SD 950-304, USA). In Biodex, the low stability level was assigned to the number 1 and the high stability level was assigned to 12 . The overall stability level is the variance of movement of the plate in relation to the degree toward the horizontal; also, the variance of the displacement of the plate in relation to the degree toward the horizontal in conjunction with the movements of the sagittal surface is considered for anterior-posterior stability level. In addition, the displacement of the plate in relation to the degree toward the horizontal in conjunction with the movements of the frontal direction is considered for the medial-lateral stability level.

The displacement of the center represents the deviation of the individual; less deviation indicates a better balance. To use the Biodex device, the participants took their shoes and socks off and they were placed on the smooth and comfortable balance meter plate. Then, looking at the device monitors, they tried to control their position. The participants performed the test at 4 states, which included standing on two legs and standing on the involved foot while the eyes are opened or closed. The plate should be fixed to measure static stability. The main test was performed in 3 repetitions and the results were reported in the form of medial-lateral, anterior-posterior, and overall stability [21] 


\section{Intervention}

The balance exercises group was treated 15 sessions for 3 weeks [6], and the duration of each session was 20 minutes; between the exercises, there was enough rest for the participants [13]. However, the strengthening exercises group was treated 15 sessions for 3 weeks and the duration of each session was 35 minutes. It should be stated that warm-up exercises were performed before the exercises of the two groups.

\section{Warm-up training}

Warm-up exercises were done for 10 minutes to prepare for exercise; the exercises were slowly walking for 5 minutes, and then stretching exercises including: 1) the patient's condition was supine while the knees were extended that in this case, the subject moved the body to the left with the opposite hand ;then, he performed it for 10 seconds and performed this exercise with the opposite hand, 2) the patient's condition was supine while one or two knees were bent over the chest, 3) the subject, then, performed hamstring muscle stretching exercises, 4) the subject performed stretching training of the adductor muscles, and 5) the subject performed stretching training of the posterior muscles of the leg.

\section{Balance exercises}

Information related to individual and medical history was checked out. In the balance group, the duration of exercises was 3 weeks and 15 sessions; each session lasted 20 minutes and there was enough rest time between each exercise [13]. The way to perform exercises was in this way:

\section{Balance training}

Keep the balance on one foot: the person should stand on one foot and should stand near a backing for support, and the other knee should be bent. The method of standing on one foot was used to measure the balance [22]. If the person was not able to maintain balance, he would stand on two feet. This procedure was also performed for the other leg and took 2 minutes [23].

The next exercise was a step that included stepping forward, back and sides for each limb; 30 repetitions were performed on each side. Along with these 30 repetitions, the next exercise was the two-way mini Scott that was performed in the painless range of 15 to 30 degrees. The aim was to strengthen the quadriceps muscle; balance exercises included the following:
1) Repeated and step-by-step exercises with 30 repetitions for the left limb; 2) 10 repetitions of two-way mini Scott; 3) Repeated and step-by-step exercises with 30 repetitions for the left limb; 4) 10 repetitions of two-way mini Scott; 5) Taking 30 steps to the left; 6) 10 repetitions of two-way mini Scott; 7) Taking 30 steps to the right [19].

\section{Strengthening exercise group}

The treatment was 5 sessions per week for 3 weeks and each session took 35 minutes [13]. In the strengthening exercise group, quadriceps muscle strength exercises were performed in sitting, standing, and supine conditions. Quadriceps muscle strength training was done in 3 sets and each set with 10 repetitions (30 repetitions in total) with low intensity [19]. There was enough interruption between exercises. The purpose of these exercises was to increase the strength of the quadriceps muscle. The level of training was easy and difficult. The duration of the training was about 35 minutes.

\section{Quadriceps muscle strength exercises in supine position:}

In this situation, a pillow is placed underneath a knee and it is flexed for a 15-degree angle. Then, the patient has to completely extend his knee and lift it up off the ground. The same process is repeated for the other knee [24].

In this situation, the patient should directly lift up his calf off the ground in an extended position. The same process is repeated for the other knee [24].

In this situation, the normal knee is bent while the involved knee is relaxed straight on the ground. Then, the involved knee is hoisted higher than the bent knee's level and it is kept in that position for 5 seconds and it will be allowed to go down smoothly. The same process is repeated for the other knee [25].

In supine status, 2 knees are on the fixed surface and the patient should move her two legs down and keep them contracted and, then, relaxed [25].

\section{Exercises in the sitting positio}

At the start of exercise, first, the knee is flexed to above 90 degrees and, then, flattened to 180 degrees that are kept contracted, and knee muscle contraction should be in a painless state and, then, slacked [19]. 


\section{Standing exercises}

The person, holding a standing position on one leg, is asked to perform 20-degree flexion exercises on the weight-bearing knee. The same process is repeated for the other knee [26].

In the standing position, the person should have twoway mini Scotts trained to increase the strength of the quadriceps without pain [19].

\section{Determining the sample size}

According to the previous studies, 26 patients with knee osteoarthritis were included in the study so that 13 patients were placed in each group.

\section{Statistical analysis}

After collecting the data, they were analyzed by SPSS 16 software. The normality of the data distribution was examined by the Kolmogorov-Smirnov test. Levene's test was used for the equality of variances. The independent t-test and paired t-test were used for inter-group and intra-group comparison, respectively. P-value was less than $5 \%$ at a significant level.

\section{Results}

Table 1 presents the average, weight, age, body mass index, and height of the two groups. Using previous reviews, the number of people participating in the study was considered 26 females for the two groups (13 females for each group). The normal distribution of the data was examined, using the Kolmogorov-Smirnov test. The results showed the normal distribution of the data (Table 1). Table 2 presents the information on stability indicators, comparison of the P-value assigned to the results of pre- and post-intervention for both groups, and the comparison of P-value assigned to results of postintervention.
Comparison of groups of balance and strengthening within the group

For balance training group, the Medial-Lateral stability Index (MLI) on the involved foot with closed eyes and the Overall Stability Index (OSI) with closed eyes on the involved foot showed a significant decrease $(\mathrm{P}<0.05)$ (Table 2). In this group, OSI with closed and opened eyes on two feet, MLI with closed and opened eyes on two feet, the Anterior-Posterior stability Index (API) with closed and opened eyes on two feet, API with the closed and opened eyes on the involved foot, MLI with opened eyes on the involved foot, and OSI with opened eyes on the involved foot reported no significant improvement (Table 2) $(\mathrm{P}>0.05)$.

For strength training group, OSI with closed and opened eyes on two feet, MLI with closed eyes on two feet, and API with closed and opened eyes on two feet showed a significant decrease $(\mathrm{P}<0.05)$. In this group, MLI with opened eyes on two feet, MLI with closed and opened eyes on the involved foot, OSI, and API reported no significant decrease (Table 2) $(\mathrm{P}>0.05)$.

\section{Comparison of groups of balance and strengthen- ing between the group}

Randomization should be checked for this purpose; the data before the study were compared in two groups. The same results were reported between the groups in the studied cases and the patients were united in the case studies in two groups. Results after treatment between groups showed no difference in the studied variables $(\mathrm{P}>0.05)$ (Table 2).

\section{Discussion}

The results of this study support the first hypothesis stating that balance exercises and strengthening exercises cause an increase in static stability indices. In addi-

Table 1. Comparing demographic characteristics between two groups

\begin{tabular}{|c|c|c|c|}
\hline \multirow{2}{*}{ Variable } & \multicolumn{2}{|c|}{ Mean $\pm S D$} & \multirow{2}{*}{ *P-value } \\
\hline & Balance Exercises $(n=13)$ & Strengthening Exercise $(n=13)$ & \\
\hline Age (y) & $49.69 \pm 9.49 *$ & $52.07 \pm 7.12$ & 0.34 \\
\hline Weight (kg) & $76.61 \pm 13.21$ & $74.38 \pm 9.74$ & 0.38 \\
\hline Height (m) & $1.56 \pm 0.09$ & $1.55 \pm 0.04$ & 0.67 \\
\hline Body mass index $\left(\mathrm{kg} / \mathrm{m}^{2}\right)$ & $31.99 \pm 8.35$ & $30.93 \pm 4.83$ & 0.60 \\
\hline
\end{tabular}

* A meaningful level of less than $5 \%$ is considered 
Table 2. Stability indices (medial-lateral, anterior-posterior, and overall) in static status

\begin{tabular}{|c|c|c|c|c|c|c|c|}
\hline \multirow{3}{*}{ Index } & \multicolumn{3}{|c|}{ (Balance Exercises) $(n=13)$} & \multicolumn{3}{|c|}{ (Strengthening Exercises) ( $n=13$ ) } & \multirow{3}{*}{$\begin{array}{l}\text { Comparing the } \\
\text { Results After } \\
\text { Intervention } \\
\text { P }\end{array}$} \\
\hline & \multicolumn{2}{|c|}{ Mean \pm SD } & \multirow{2}{*}{$\mathbf{P}$} & \multicolumn{2}{|c|}{ Mean $\pm S D$} & & \\
\hline & Pre- treatment & Post- treatment & & Pre- treatment & Post- treatment & & \\
\hline SMOSIBEO & $* 40.0 \pm 0.37$ & $0.55 \pm 0.71$ & 0.06 & $1.26 \pm 0.82$ & $0.67 \pm 0.84$ & $0.20 *$ & 0.16 \\
\hline SMOSIBEC & $2.13 \pm 1.03$ & $1.37 \pm 0.27$ & 20.0 & $3.35 \pm 1.75$ & $00.1 \pm 34.2$ & $0.10^{*}$ & 0.09 \\
\hline SMAPIBEO & $0.55 \pm 0.36$ & $0.83 \pm 0.21$ & 0.06 & $0.78 \pm 0.53$ & $0.65 \pm 0.73$ & $0.20 *$ & 0.11 \\
\hline SMAPIBEC & $1.63 \pm 1.07$ & $1.13 \pm 0.27$ & 0.32 & $2.88 \pm 1.77$ & $1.99 \pm 1.50$ & $0.20 *$ & 0.06 \\
\hline SMMLIBEO & $0.31 \pm 0.12$ & $0.23 \pm 0.13$ & 0.77 & $0.66 \pm 0.74$ & $0.93 \pm 0.92$ & 0.02 & 0.45 \\
\hline SMMLIBEC & $1.00 \pm 50.0$ & $0.38 \pm 0.45$ & 0.61 & $1.24 \pm 0.46$ & $0.98 \pm 0.73$ & $0.20^{*}$ & 0.70 \\
\hline SMOSIUSIEO & $8.23 \pm 3.15$ & $7.55 \pm 2.93$ & 0.91 & $9.00 \pm 2.86$ & $8.59 \pm 2.89$ & 0.78 & 0.24 \\
\hline SMOSIUSIEC & $9.51 \pm 3.18$ & $7.35 \pm 2.81$ & $00.00 *$ & $9.28 \pm 3.69$ & $8.96 \pm 2.53$ & 0.33 & 0.26 \\
\hline SMAPIUSIEO & $2.26 \pm 1.16$ & $1.79 \pm 1.50$ & 0.35 & $2.11 \pm 1.54$ & $1.18 \pm 1.63$ & 0.83 & 0.77 \\
\hline SMAPIUSIEC & $3.17 \pm 1.16$ & $2.06 \pm 1.22$ & 0.62 & $3.71 \pm 2.25$ & $2.64 \pm 1.66$ & 0.62 & 0.82 \\
\hline SMMLIUSIEO & $7.78 \pm 3.28$ & $7.30 \pm 2.91$ & 0.02 & $8.18 \pm 3.10$ & $8.95 \pm 3.00$ & 0.33 & 0.19 \\
\hline SMMLIUSIEC & $8.27 \pm 3.32$ & $6.77 \pm 2.95$ & $00.00 *$ & $8.34 \pm 3.35$ & $8.40 \pm 2.44$ & 0.15 & 0.24 \\
\hline
\end{tabular}

* A meaningful level of less than $5 \%$ is considered.

Iranian Rehabilitation Journa

THE comparison of the P-value assigned to the results of pre- and post-intervention for two groups, and the comparison of $\mathrm{P}$-value assigned to the results post-intervention.

tion, unlike the second hypothesis, there was no difference between the two treatment methods in terms of all static stability indices.

The results of our study revealed that balance exercises improved MLI with closed eyes on the involved foot and OSI with closed eyes on the involved foot (Table 2). However, these exercises had no impact on MLI with closed and opened eyes on two feet, OSI with closed and opened eyes on two feet, API with closed and opened eyes on two feet, MLI with opened eyes on the involved foot, OSI with opened eyes on the involved foot, and API with closed and opened eyes on the involved foot (Table 2).

The strengthening exercises group improved OSI with closed and opened eyes on two feet, MLI with closed eyes on two feet, and API with closed and opened eyes on two feet. In addition, it had no impact on MLI with opened eyes on two feet and MLI, OSI, and API with closed and opened eyes on the involved foot (Table 2). No difference was found between the two treatment groups while strengthening exercises improved stability indices more than that in the balance exercises group.

In a study carried out by Shah Hosseini et al. in 2004, two therapeutic programs, muscle strengthening and neuromuscular control rehabilitation, were compared in terms of their effects on the improvement of the treatment indices in patients with the osteoarthritic knee [14]. The results of their study are consistent with our study; both of the studies reported improvements in balance indices of the muscle-strengthening group and the neuromuscular control rehabilitation group; in addition, it was found out that the improvement in the postural stability indices can be attributed to the following cases: the muscle strengthening program causes corrections in the motor part of the quadriceps motor-sensory function disorder and there is a need for taking advantage of neuromuscular control exercises to correct the sensory part. Thus, this new treatment method that incorporated the modulation of both sensory and motor parts was found more effective in the improvement of proprioception than the common treatment method only emphasizing the reformation of the motor part [14]. 
Common exercise therapy that underlines the strengthening of quadriceps causes improvement in one of the main factors of balance control to wit the muscular strength, but the new exercise therapy that encompasses the neuromuscular rehabilitation exercises in addition to the strengthening exercise causes improvement in the muscular strength and proprioception of these patients. This is indicative of the effective role of these exercises on balance improvement [14]. Contrarily, the findings of the present study do not confirm the results obtained by Lavand et al.'s research, in which 8 weeks of training in water and on land did not have any significant effect on the balance of the individuals with knee arthritis; whereas, there was a significant improvement in the group that was only trained on land in terms of muscle strength [2].

In a study performed by Knop et al., a home-based exercise protocol was applied that embraced muscle strengthening exercises and balance exercises, which was performed by 159 patients with knee instability and also by the patients with the osteoarthritic knee for a period of 12 weeks [27]. Their results were in compliance with the results of the present study in that the improvement in stability was evidenced in both of the groups. The improvement can be a result of the fact that the muscle strengthening exercises reduced the joint pain that led to improvement in performance and stability [28].

Kyung Kim et al. reported in 2016 that patients were assigned to two groups, i.e. muscle strength training and strengthening-balance exercises [29]. The results were in accordance with the results of our study in that a significant improvement was not reported between the two groups, but an increase in postural stability and a reduction in pain intensity was reported in both of the groups [29].

The results indicated that both of the exercise groups stimulated the proprioception, which was effective in knee stability elevation. Moreover, the increase in stability brought about a reduction in the related knee pain [29]. Holden et al. found no improvement in the balance of the patients with knee osteoarthritis that can be following treatment method of study [30].

The reason why the static stability indices have been improved following taking the balance and musclestrengthening programs of quadriceps might be explained as recounted in the following statements. The improvement in static stability found in the present study can be attributed to the effects of exercise therapy like the physiological effects, muscle strengthening, and neuromuscular coordination [31]. Since the individuals with knee osteoarthritis are usually found with muscular weakness in quadriceps, as well as hamstring and disorders in neuromuscular coordination, the balance and strength training along with neuromuscular improvement and strengthening of the knee joint muscles can result in improvement in balance [16].

It seems that the strengthening of the quadriceps and hamstring muscles is the reason why the static stability of these patients has been improved [16]. The issue can justify the improvement in many of the indices in the strength training group compared to the balance training group. Also, with regard to the effect of balance exercises on the static stability, it can be stated that the preservation of balance entails the cooperation among 3 aspects of information processing by visual, vestibular and somatosensory systems, central integration of the brain, and motor response.

Any malfunctioning in this neuromuscular system can be a risk factor giving rise to the falls. Considering the fact that every individual is helped by the visual system, vestibular system, and somatosensory system to stay balanced, it is worth mentioning that with regard to static stability evaluations, the balance exercises by the use of neuromuscular system causes improvement and facilitation of the inputs to each of these senses so as to preserve postural balance. Therefore, the possible reasons behind the static balance improvement can be sought in the increase in the neural adaptations resulting from exercises such as the use of more efficient neural units, rearrangement of the somatosensory pathways, increase in synoptic efficiency, improvement in neuromuscular control, muscular strengthening, and facilitation of the input transmission from each of these senses [32].

According to the above-mentioned cases, it can be asserted that balance exercises and strength training of the quadriceps in women with knee osteoarthritis are effective in improving the static stability indices. Quadriceps strength training causes improvement in more stability indices, but no significant difference was found between the two groups that this is probably because of the short duration of the exercises. Future studies with a longer period of examination time, as well as follow-up tests, are suggested. Another limitation of the current research was the low volume of the study participants; hence, further research can be carried out on a larger sample volume.

\section{Conclusion}

The results of the present study indicated two treatment groups reporting the same results with regard to the ef- 
fect on static stability indices. But, the strength training causes improvement in a greater number of the postural stability indices in contrast to the balance exercises.

\section{Ethical Considerations}

Compliance with ethical guidelines

This study was registered (No.IR.ZAUMS. REC.1395.226) and approved by the Scientific and Ethics Committee of the Rehabilitation Department of Zahedan University of Medical Sciences. After reading and signing a written informed consent form, the participants were included in the study protocol.

Funding

This article was the result of the project in the Department of Physiotherapy, School of Rehabilitation Sciences, Zahedan University of Medical Sciences.

\section{Authors' contributions}

Conception, design, and writing: Narges Jahantigh Akbari; Planning: Ahmad Reza Askary Ashtiani; Implementation of intervention: Narges Jahantigh Akbari and Ahmad Reza Askary Ashtiani; and Helping in writing: Salman Nouraisarjou.

\section{Conflict of interest}

The authors declared no conflict of interest.

\section{References}

[1] Rozzi S, Yuktanandana P, Pincivero D, Lephart S. Role of fatigue on proprioception and neuromuscular control. Proprioception and Neuromuscular Control in Joint Stability Champaign, IL: Human Kinetics. 2000; 2000:375-83.

[2] Lund H, Weile U, Christensen R, Rostock B, Downey A, Bartels EM, et al. A randomized controlled trial of aquatic and land-based exercise in patients with knee osteoarthritis. Journal of Rehabilitation Medicine. 2008; 40(2):137-44 [DOI:10.2340/16501977-0134] [PMID]

[3] Song R, Lee E-O, Lam P, Bae S-C. Effects of tai chi exercise on pain, balance, muscle strength, and perceived difficulties in physical functioning in older women with osteoarthritis: A randomized clinical trial. The Journal of Rheumatology. 2003 30(9):2039-44

[4] Hinman R, Bennell K, Metcalf B, Crossley K. Balance impairments in individuals with symptomatic knee osteoarthritis: A comparison with matched controls using clinical tests. Rheu- matology. 2002; 41(12):1388-94. [DOI:10.1093/rheumatology/41.12.1388] [PMID]

[5] Masui T, Hasegawa Y, Yamaguchi J, Kanoh T, Ishiguro $\mathrm{N}$, Suzuki S. Increasing postural sway in rural-communitydwelling elderly persons with knee osteoarthritis. Journal of Orthopaedic Science. 2006; 11(4):353-8. [DOI:10.1007/s00776006-1034-9] [PMID]

[6] Lyytinen T, Liikavainio T, Bragge T, Hakkarainen M, Karjalainen PA, Arokoski JP. Postural control and thigh muscle activity in men with knee osteoarthritis. Journal of Electromyography and Kinesiology. 2010; 20(6):1066-74. [DOI:10.1016/j. jelekin.2010.05.005] [PMID]

[7] Peat G, McCarney R, Croft P. Knee pain and osteoarthritis in older adults: A review of community burden and current use of primary health care. Annals of the Rheumatic Diseases. 2001; 60(2):91-7. [DOI:10.1136/ard.60.2.91] [PMID] [PMCID]

[8] Fisher N, Pendergast D. Reduced muscle function in patients with osteoarthritis. Scandinavian Journal of Rehabilitation Medicine. 1997; 29(4):213-21.

[9] Sharma L. Proprioceptive impairment in knee osteoarthritis. Rheumatic Disease Clinics. 1999; 25(2):299-314. [DOI:10.1016/ S0889-857X(05)70069-7]

[10] Hurley MV, Scott DL, Rees J, Newham DJ. Sensorimotor changes and functional performance in patients with knee osteoarthritis. Annals of the Rheumatic Diseases. 1997; 56(11):641-8. [DOI:10.1136/ard.56.11.641] [PMID] [PMCID]

[11] Hughes MA, Duncan PW, Rose DK, Chandler JM, Studenski SA. The relationship of postural sway to sensorimotor function, functional performance, and disability in the elderly. Archives of Physical Medicine and Rehabilitation. 1996; 77(6):567-72. [DOI:10.1016/S0003-9993(96)90296-8]

[12] Diracoglu D, Aydin R, Baskent A, Celik A. Effects of kinesthesia and balance exercises in knee osteoarthritis. JCR: Journal of Clinical Rheumatology. 2005; 11(6):303-10. [DOI:10.1097/01.rhu.0000191213.37853.3d] [PMID]

[13] Duman I, Taskaynatan MA, Mohur H, Tan AK. Assessment of the impact of proprioceptive exercises on balance and proprioception in patients with advanced knee osteoarthritis. Rheumatology international. 2012; 32(12):3793-8. [DOI:10.1007/s00296-011-2272-5] [PMID]

[14] Shah Hosseini G, Negahban Siuki H, Madani S, Ebrahimi Takamjani E, Shaterzadeh M. [Comparison of the effect of two therapeutic methods (traditional \& new) on therapeutic parameters in patients with primary knee osteoarthritis (Persian)]. Razi Journal of Medical Sciences. 2004; 10(37):735-41.

[15] Perlau R, Frank C, Fick G. The effect of elastic bandages on human knee proprioception in the uninjured population. The American Journal of Sports Medicine. 1995; 23(2):251-5. [DOI: 10.1177/036354659502300221] [PMID]

[16] Ezadpanah A, Moazami M, Khoshraftar Yazdi N. [Effect of a period of therapeutic exercise and detraining after that on balance in the women with knee osteoarthritis(Persian)]. Journal of Modern Rehabilitation. 2016; 9(5):101-9.

[17] Rätsepsoo M, Gapeyeva H, Sokk J, Ereline J, Haviko T, Pääsuke M. Leg extensor muscle strength, postural stability, and fear of falling after a 2-month home exercise program in women with severe knee joint osteoarthritis. Medicina 
(Kaunas, Lithuania). 2013; 49(8):347-53. [DOI:10.3390/medicina49080054]

[18] Felson DT, Zhang Y, Hannan MT, Naimark A, Weissman $\mathrm{BN}$, Aliabadi $\mathrm{P}$, et al. The incidence and natural history of knee osteoarthritis in the elderly, the framingham osteoarthritis study. Arthritis \& Rheumatology. 1995; 38(10):1500-5. [DOI:10.1002/art.1780381017] [PMID]

[19] Chaipinyo K, Karoonsupcharoen O. No difference between home-based strength training and home-based balance training on pain in patients with knee osteoarthritis: A randomised trial. Australian Journal of Physiotherapy. 2009; 55(1):25-30. [DOI:10.1016/S0004-9514(09)70057-1]

[20] Kim HS, Yun DH, Yoo SD, Kim DH, Jeong YS, Yun JS, et al. Balance control and knee osteoarthritis severity. Annals of Rehabilitation Medicine. 2011; 35(5):701-9. [DOI:10.5535/ arm.2011.35.5.701] [PMID] [PMCID]

[21] Akbari A, Ghiasi F, Mir M, Hosseinifar M. The effects of balance training on static and dynamic postural stability indices after acute ACL reconstruction. Global Journal of Health Science. 2016; 8(4):68. [DOI:10.5539/gjhs.v8n4p68] [PMID] [PMCID]

[22] Kavounoudias A, Gilhodes J-C, Roll R, Roll J-P. From balance regulation to body orientation: Two goals for muscle proprioceptive information processing? Experimental Brain Research. 1999; 124(1):80-8. [DOI:10.1007/s002210050602] [PMID]

[23] McCarthy C, Mills P, Pullen R, Richardson G, Hawkins N Roberts C, et al. Supplementation of a home-based exercise programme with a class-based programme for people with osteoarthritis of the knees: A randomised controlled trial and health economic analysis. Health Technology Assessment (Winchester, England). 2004; 8(46): 1-61. [DOI:10.3310/ hta8460] [PMID]

[24] Røgind H, Bibow-Nielsen B, Jensen B, Møller HC, FrimodtMøller $\mathrm{H}$, Bliddal $\mathrm{H}$. The effects of a physical training program on patients with osteoarthritis of the knees. Archives of Physical Medicine and Rehabilitation. 1998; 79(11):1421-7. [DOI:10.1016/S0003-9993(98)90238-6]

[25] Shakoor N, Furmanov S, Nelson D, Li Y, Block J. Pain and its relationship with muscle strength and proprioception in knee OA: Results of an 8-week home exercise pilot study. Journal of Musculoskeletal \& Neuronal Interactions. 2008; 8(1):35-42.

[26] Kiss RM. Effect of severity of knee osteoarthritis on the variability of gait parameters. Journal of Electromyography and Kinesiology. 2011; 21(5):695-703. [DOI:10.1016/j.jelekin.2011.07.011] [PMID]

[27] Knoop J, Dekker J, Van der Leeden M, Van der Esch M, Thorstensson C, Gerritsen M, et al. Knee joint stabilization therapy in patients with osteoarthritis of the knee: A randomized, controlled trial. Osteoarthritis and Cartilage. 2013; 21(8):1025-34. [DOI:10.1016/j.joca.2013.05.012] [PMID]

[28] Henriksen M, Rosager S, Aaboe J, Graven-Nielsen T, Bliddal $\mathrm{H}$. Experimental knee pain reduces muscle strength. The Journal of Pain. 2011; 12(4):460-7. [DOI:10.1016/j. jpain.2010.10.004] [PMID]

[29] Kim K, Lee HY, Lim SJ. Effects of increased standing balance on pain in patients with knee osteoarthritis. Journal of physical therapy science. 2016; 28(1):87-9. [DOI:10.1589/ jpts.28.87] [PMID] [PMCID]

[30] Holden MA, Nicholls EE, Young J, Hay EM, Foster NE. UK-based physical therapists' attitudes and beliefs regarding exercise and knee osteoarthritis: Findings from a mixedmethods study. Arthritis Care \& Research. 2009; 61(11):151121. [DOI:10.1002/art.24829] [PMID]

[31] Akbar M. Therapeutic exercise. The University of Guilan. 2005.

[32] Stevens JA, Olson S. Reducing falls and resulting hip fractures among older women. Home Care Provider. 2000; 5(4):134-41. [DOI:10.1067/mhc.2000.109232] [PMID] 
This Page Intentionally Left Blank 